\title{
A Medium Model for the Refrigerant Propane for Fast and Accurate Dynamic Simulations
}

\author{
Roozbeh Sangi \\ Pooyan Jahangiri \\ Freerk Klasing \\ Rita Streblow Dirk Müller \\ Institute for Energy Efficient Buildings and Indoor Climate, \\ E.ON Energy Research Center, RWTH Aachen University, \\ Mathieustr.10, 52074 Aachen, Germany \\ rsangi@eonerc.rwth-aachen.de
}

\begin{abstract}
Investigating the use of different fluids and their advantages in new energy systems has increased the need for faster and more robust simulation models. The need to explore the potential of new fluids in different systems requires dynamic simulations for longer periods of time. In this paper, developing and improving a medium model for propane is discussed. Besides being fast and accurate, the propane model should also be stable in different dynamic simulation scenarios.

First, existing libraries are tested and in some cases modified to increase the stability. Since the simulation speeds were not in an acceptable range in the existing models, a new propane model based in the refrigerant models in HelmholzMedia Library is introduced. The new model is then tested as a refrigerant in a direct exchange heat pump system. A comparison between an existing propane model and the new model shows that much faster simulations, up to 35 times, are possible with the new propane model.
\end{abstract}

Keywords: Media Library; Propane; Refrigerant; Helmholtz Media

\section{Introduction}

To address the main challenges arising from an ever increasing energy demand worldwide and its associated environmental impacts, it is not only essential to optimize the existing energy landscape but is also necessary to develop new approaches. One of these approaches is to utilize low-exergy heating and cooling systems.

A well-known method is by the use of heat pumps to extract energy stored in the ground. A conventional combination of a heat pump and a heat- ing/cooling unit consists of three different hydraulic cycles, a primary cycle (energy source), a secondary cycle (energy sink) and a refrigerant cycle in between. For dynamic analysis of a complete heating or cooling system using a heat pump, the refrigerant cycle is usually considered as a black box model. This means that the thermal behavior of the refrigerant under different conditions is considered to be known without knowing the actual state of the refrigerant. Although this will reduce the accuracy of such a simulation, in larger scales, its great impact in the simulation speed is of higher importance.

To improve the affordability of heat pumps and reduce thermal losses in the heat exchangers between each cycle, the concept of direct exchange heat pumps is considered. This means that instead of having a heat exchanger between the energy source (ground) and the refrigerant cycle, the refrigerant flows directly inside the ground source. In simulations of such systems, a black-box model cannot be used and the whole behavior of the refrigerant should be simulated without compromising the simulation speed.

There are different methods of simulating different refrigerants. C. Heinrich et al. [1] have developed models for household refrigerant applicances using R600a, T. Pfafferott et al. [2, 3] have developed refrigerant models with $\mathrm{CO}_{2}$ and R134a and I. Bell et al. [4] have created an open-source fluid library available for many different platforms using the Helmholz equation of state. These methods use either internal or external functions. Although these methods can be expanded to different refrigerants, a separate propane model is not developed and the accuracy of the models was the main subject and not the simulation speed. Since simulation speed is of great concern, external media functions are not considered in this study. In the next chapter an available method of describing the behavior of refrigerants 
internally is discussed. For further simplifications, only one refrigerant is considered.

Because of its low environmental impact, propane is chosen as the refrigerant and the modeling of this refrigerant is described in this paper.

\section{Available Refrigerant Model}

The design and simulation of heat pumps or power cycles require an accurate representation of the working fluid. In general a fluid is described by the equation of state and additional transport properties. The equation of state defines all thermodynamic properties in terms of two independent thermodynamic state variables. Usually these two variables are pressure $(p)$ and enthalpy $(h)$, temperature $(T)$ and density $(\rho)$ or, in case of one phase only, pressure and temperature. Today fundamental equations of state (EoS) in terms of Helmholtz energy are the most accurate method available for this purpose [5].

\subsection{Helmholtz Energy Equation of State}

As described in detail by [6], the Helmholtz EoS uses temperature and specific volume or density of the fluid as the independent variables. Other variables such as specific enthalpy, specific entropy as well as partial derivatives of several thermodynamic state variables can be calculated directly from these independent variables. Additional ancillary equations express the vapor pressure and the saturated densities in terms of the temperature and thereby define the boundary between single-phase and two-phase. Transport properties like surface tension, viscosity and thermal conductivity however are not part of the equations of state and have to be supplied for with additional independent correlations.

The Helmholtz energy equation of state excludes the two-phase region. Here all the remaining properties can be calculated under the condition of mechanical and chemical equilibrium for the liquid and the vapor phase. With this assumption, all thermodynamic properties can be calculated as a function of the dew and bubble state properties and the resulting steam quality.

A comprehensive explanation of the Helmholtz energy equation of state for the refrigerant propane can be found in [7].

\subsection{HelmholtzMedia Fluid Properties Library}

The HelmholtzMedia Fluid Properties Library is an open-source Modelica library. The library is devel- oped to be expandable and contains several different refrigerants. The library covers a wide variety of applications since the implemented EoS are valid for a wide range of state variables.

As mentioned in [6], since the EoS are implemented in their general form, the library is not optimized for speed. Also because the fluid library is designed for all conditions, it may be unstable in certain complex combination of different components, such as complex energy systems that include closed fluid circuits, which should be avoided in large scale simulations.

\section{Methods of Calculation of EoS}

\subsection{Inverse Calculation of EoS}

Common variable combinations for engineering applications often are $(p, T),(p, h)$ or $(p, s)$. The reason for choosing these independent state variables is on the one hand an improved computational stability and on the other hand the fact that changes of state are usually expressed via these variables. There are two ways to calculate the inverse of the equations of state. The first way is to use an implicit description which was done in the HelmholtzMedia Fluid Properties Library. The second solution is to do it explicitly which is done in the new propane model discussed later on.

\subsection{Implicit Calculation of Inverse EoS}

In order to compute all other thermodynamic properties from the chosen state variables, the corresponding $(T, \rho)$ must then be determined iteratively, since the fundamental equation of state in terms of Helmholtz energy is non-reversible.

The iteration is a root finding algorithm available in the base library of Modelica. The two-phase boundary of the fluid is described by the vapor liquid equilibrium. The dew and bubble states are then calculated by solving two out of three conservative equations: the thermal equilibrium, the mechanical equilibrium and the chemical equilibrium. Ancillary equations for the saturation pressure and the dew and bubble densities help finding these states giving start values for the iteration. They also serve for a first region check for the inverse equation of state calculation. In the close proximity to the saturation line however, the exact values must be calculated by solving the vapor-liquid-equilibrium. The vaporliquid-equilibrium represents a state where the rate of evaporation and the rate of condensation are the same on a molecular level. It is characterized by 
three conditions: thermal equilibrium, mechanical equilibrium and chemical equilibrium [8].

\subsection{Explicit Calculation of Inverse EoS}

Due to the high computational effort for the implicit calculation of state an alternative way for expressing the state variables in terms of $(p, T),(p, h)$ or $(p, s)$ is implemented in Modelica. For these calculations, polynomial fit functions were developed for a predefined region. The maximum and minimum values were chosen according to the approximate range of operation of the application. Here a temperature region between $-10^{\circ} \mathrm{C}$ and $70^{\circ} \mathrm{C}$ and a pressure region between 0.5 bars and 30 bars was chosen (see Figure 1).

For this purpose, the inverse of the whole EoS was calculated numerically. The resulted data-set was then divided into six different sets (sub cooled liquid and superheated steam for density, specific enthalpy and specific entropy). Then a surface was fitted to each data-set separately. The outcomes of this fitting were six polynomial functions of order $5 \times 5$.

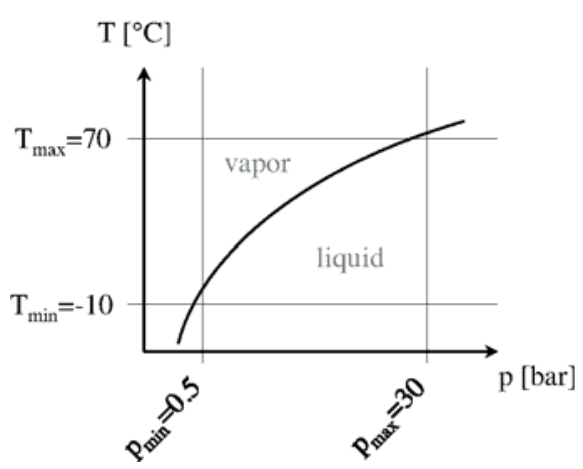

Figure 1: Range of validity for the inverse EoS

\section{Implementation in Modelica}

For all the necessary parameters, surface equations are added to the new media library. An example of such a surface is illustrated in Figure 2.The implemented media library is an extension of PartialTwoPhaseMedium which itself is an extension of PartialMedium in the Modelica Standard Library. This means that the simple propane model is also compatible with all the existing components in the Fluid library.

Moving from one region to the other makes the system not continues resulting in instabilities in solving the system of differential equations and therefore the whole simulation. To avoid these certain functions are developed for the smooth transition between each state of the fluid. These functions not only include the correct value for the properties of propane at the discontinuous point, but also include the derivative so that smooth transitions are possible. Although this method will improve stability, since it is only applied in one point, the discontinuity of the functions can still be observed in the results.

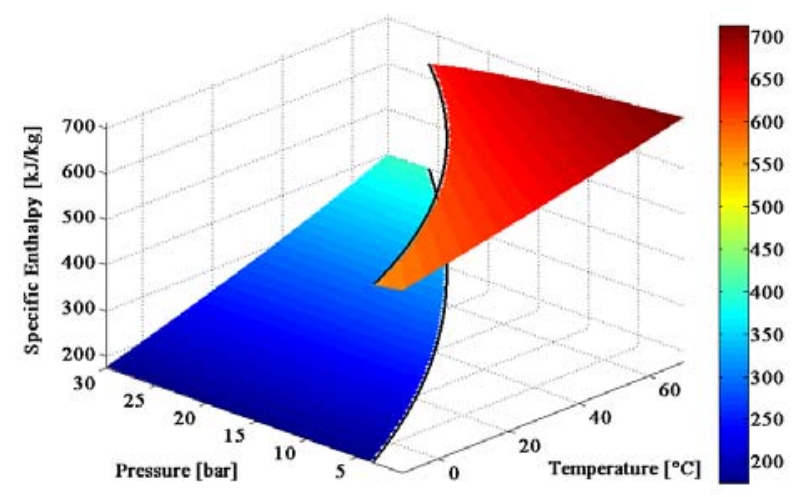

Figure 2: Illustration of the fitted surfaces in a predefined region for pressure, temperature and specific enthalpy

\section{Results and Discussion}

\subsection{Accuracy of the Fitted Functions}

As discussed earlier, it is important for the model to be accurate. For this purpose, the Sum of squared errors (CSE), coefficient of determination (RSQUARE), number of evaluated points (DFE) and root mean square error (RMSE) are calculated for the fitted curves and are shown in Table 1. The low error values indicate a very fine fitting of the data.

Table 1: Accuracy of the inverse equations

\begin{tabular}{ccccc}
\hline Function & SSE & RSQUARE & DFE & RMSE \\
\hline$\rho(p, T)_{G}$ & 0.009132 & 1.000000 & 296656 & $1.7545 \mathrm{e}-4$ \\
$\rho(p, T)_{L}$ & 0.185102 & 1.000000 & 104686 & 0.001330 \\
& & & & \\
$T(p, h)_{G}$ & 0.073031 & 1.000000 & 296656 & $4.9616 \mathrm{e}-4$ \\
$T(p, h)_{L}$ & $2.2526 \mathrm{e}-4$ & 1.000000 & 104686 & $4.6387 \mathrm{e}-5$ \\
& & & & \\
$T(p, s)_{G}$ & 1.171992 & 1.000000 & 296656 & 0.001988 \\
$T(p, s)_{L}$ & $7.9326 \mathrm{e}-4$ & 1.000000 & 104642 & $8.7067 \mathrm{e}-5$ \\
\hline
\end{tabular}

For comparison between the HelmholtzMedia and the fast propane model, both fluid models are simulated at different temperature and pressures in Modelica. The relative error between the two simulations is then calculated and is shown in Table 2. The relative error in all cases is close to zero. For all the simulation parameters, this error is much smaller than the uncertainty in the system. 
Table 2: Relative error between propane in HelmholtzMedia and the fast propane

\begin{tabular}{ccrrrr}
\hline $\boldsymbol{T}$ & $\begin{array}{c}\boldsymbol{\rho} \\
\mathrm{mol} / \mathrm{cm}^{3}\end{array}$ & $\begin{array}{c}\boldsymbol{p} \\
\text { Rel. Err. }\end{array}$ & $\begin{array}{c}\boldsymbol{C}_{\boldsymbol{V}} \\
\text { Rel. Err. }\end{array}$ & $\begin{array}{c}\boldsymbol{C}_{\boldsymbol{P}} \\
\text { Rel. Err. }\end{array}$ & $\begin{array}{c}\boldsymbol{w} \\
\text { Rel. Err. }\end{array}$ \\
\hline 200.0 & 14.0 & $-2.1 \mathrm{e}-8$ & $3.9 \mathrm{e}-8$ & $2.9 \mathrm{e}-8$ & $1.4 \mathrm{e}-10$ \\
300.0 & 12.0 & $-5.2 \mathrm{e}-8$ & $1.0 \mathrm{e}-7$ & $5.0 \mathrm{e}-8$ & $1.3 \mathrm{e}-8$ \\
300.0 & 0.4 & $3.2 \mathrm{e}-8$ & $8.8 \mathrm{e}-8$ & $9.4 \mathrm{e}-9$ & $-1.2 \mathrm{e}-8$ \\
400.0 & 5.0 & 0.0 & $1.9 \mathrm{e}-8$ & $-2.0 \mathrm{e}-8$ & $-3.8 \mathrm{e}-8$ \\
369.9 & 5.0 & $2.4 \mathrm{e}-8$ & $6.0 \mathrm{e}-8$ & 0.0 & $-3.8 \mathrm{e}-8$ \\
\hline
\end{tabular}

It can be seen that in the specified range, the relative error between two models are very close to zero. This means if the change in propane properties does not fall behind the boundaries chosen for the surfacefitting, both models can be used interchangeably with minimal compromise in accuracy.

\subsection{Simulation Speed Comparison}

Since water is the main fluid used in different energy systems, simulations with water can be set as base simulations for comparison of the simulation time for the new fluid models. Here, the simulation speed of both fluid models are compared in identical simulation models with a reference model with the simple water model available in Modelica standard library as its medium. The results are shown in Figure 3.

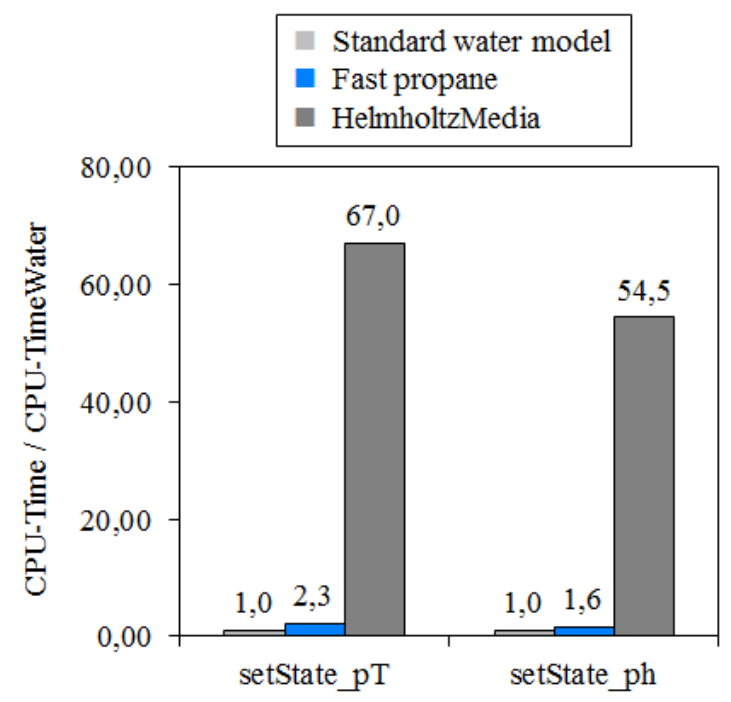

Figure 3: Simulation times for different propane models relative to water

The simulation speed of the fast propane is around 2 times slower than that of water but it is 30 times faster than the complete Propane model from the HelmholzFluid library. This increase in simulation speed can ensure the possibility of simulating complete energy systems for a much longer period of time (monthly or yearly simulations).

\section{Conclusions}

To increase the stability and decrease the simulation time, HelmholtzMedia model for propane is modified for a certain region. The inverse calculation of the equations of states is done using several fitted surfaces instead of the actual EoS for the calculation of different variables.

It can be observed that the relative error between both models is almost zero, and on the other hand a significant increase in the simulation speed can be achieved, making the use of the fast propane model for larger simulations possible.

To conclude the differences between the two models, a simple comparison between the two models is shown in Table 3. This shows that although the HelmholzMedia can be used for much wider range applications, for certain applications (ranges) it can be simplified so that much faster and more stable simulations are possible.

Table 3: Comparison between HelmholtzMedia and fast propane model

\begin{tabular}{lcc}
\hline Property & Helmholtz Media & Fast Propane \\
\hline $\begin{array}{l}\text { Stability in } \\
\text { complex systems }\end{array}$ & $\begin{array}{c}\text { Not fully stable in } \\
\text { complex energy } \\
\text { systems with } \\
\text { closed fluid cir- } \\
\text { cuits }\end{array}$ & $\begin{array}{c}\text { Fully Stable between } \\
-10^{\circ} \mathrm{C} \text { and } 70^{\circ} \mathrm{C} \text { and } \\
\text { between } 0.5 \text { to } 30 \\
\text { bars }\end{array}$ \\
$\begin{array}{l}\text { Accuracy } \\
100 \%\end{array}$ & $\begin{array}{c}100 \% \text { between }-10^{\circ} \mathrm{C} \\
\text { and } 70^{\circ} \mathrm{C} \text { and be- } \\
\text { tween } 0.5 \text { to } 30 \text { bars }\end{array}$ \\
$\begin{array}{l}\text { Simulation } \\
\text { tion speed for } \\
\text { simple water }\end{array}$ & $60 \mathrm{x}$ & $2 \mathrm{x}$ \\
$\begin{array}{l}\text { Number of } \\
\text { available media }\end{array}$ & & 1 \\
\hline
\end{tabular}

The downside of the fast propane model is that it is only valid for a certain range of temperatures and pressures. Although this range is sufficient for many engineering applications in low temperature and low exergy heating and cooling systems, it is not sufficient for all the applications.

\section{References}

[1] C. Heinrich, K. Berthold "A Modelica Library for Simulation of Household Refrigeration Appliances Features and Experiences”. In: Proceedings of the $5^{\text {th }}$ International Modelica Conference, 2006, pp. 677-684. 
[2] T. Pfafferott, G. Schmitz "Modeling and Simulation of Refrigeration Systems with the Natural Refrigerant CO2" In: Proceesings of the International Refrigeration and Air Conditioning Conference, 2002.

[3] T. Pfafferott, G. Schmitz “ Implementation of a Modelica Library for Simulation of Refrigeration Systems" In: Proceedings of the $3^{\text {rd }}$ International Modelica Conference, 2003, pp. 197-206.

[4] I. Bell, S. Quoilin, J. Wronski, V. Lemort "CoolProp: An Open-Source ReferenceQuality Thermophysical Property Library" In: $2^{\text {nd }} A S M E$ ORC International Seminar, 2013.

[5] M. Thorade, A. Saadat "Partial Derivatives of Thermodynamic State Properties for Dynamic Simulation”. In: Environmental Earth Sciences (online first), 2012. DOI: 10.1007/s12665-013-2394-z.

[6] M. Thorade, A. Saadat "HelmholtzMedia A Fluid Properties Library”. In: Proceedings of the $9^{\text {th }}$ International Modelica Conference, 2012, pp. 63-69. DOI: 10.3384/ecp1207663

[7] E. W. Lemmon, M. O. McLinden, W. Wagner "Thermodynamic Properties of Propane. III. A Reference Equation of State for Temperature from Melting Line to $650 \mathrm{~K}$ and Pressures up tp 1000 MPa”. In: Journal of Chemical Engineering Data 54, 2009, pp. 3141-3180.

[8] K. Lucas, "Thermodynamik, Die Grundgesetze der Energie- und Stoffumwandlungen”, Sprinter Verlag Berlin, 7. Edition, 2008. ISBN-13: 9783540686453 